\title{
La douleur n'a sans doute pas de neurotransmetteur spécifique dans la moelle épinière
}

De nombreux neuropeptides ont été identifiés dans les circuits impliqués dans la transmission et le contrôle des messages liés à la nociception et à la douleur [1, 2]. Parmi eux, deux types de peptides avaient les honneurs du plus large public : les enképhalines et la substance $P$.

Pour les premières, le cas était, semble-t-il, réglé puisque c'est parce qu'ils se fixaient sur les mêmes récepteurs que la morphine qu'ils avaient été identifiés. Nul doute que ces morphiniques endogènes devaient jouer un rôle dans le contrôle - le blocage - des influx nerveux liés à la douleur. Las! l'histoire s'est compliquée au cours des années et nul ne prétend plus aujourd'hui que les enképhalines - ou une quelconque des autres «endomorphines », puisque la famille s'est considérablement agrandie - sont spécifiquement impliquées dans les mécanismes nerveux liés à la douleur. Elles agissent, de façon variée, dans un très grand nombre de sites cérébraux et, très vraiser.sblablement, sur un grand nombre de fonctions dont bien peu, c'est une évidence, sont directement liées à la douleur.

Pour la substance $P$, le rôle de « neurotransmetteur de la douleur » dans la moelle épinière est resté peu contesté jusqu'à aujourd'hui. On trouve, en effet, de la substance $P$ dans un grand nombre des petites cellules des ganglions rachidiens à l'origine des fibres non myélinisées - dites de type $\mathrm{C}-$ qui jouent, sans aucun doute, un rôle primordial dans la transmission des messages liés à la thermonociception (douleur provoquée par la température), et important dans la mécanonociception [3]. Les résultats électrophysiologiques semblaient bien indiquer une action facilitatrice de la substance $P$ sur les neurones « nociceptifs » de la moelle épinière [4], cependant le décours des $\mathrm{m} / \mathrm{s} n^{\circ} 3$ vol. 5 , mars 89 effets du peptide sur l'activité neuronale semblait trop lent pour correspondre à l'action attendue d'un véritable neurotransmetteur [5]. Il y avait, d'autre part, un problème avec les fibres non myélinisées qui convoient les messages de la douleur viscérale, car elles ne contiennent pas de substance $P$. Une solution avai cependant été trouvée : elles contiennent du VIP (vasoactive intestinal peptide), qui fut donc étiqueté neurotransmetteur de la douleur viscérale [5].

Les résultats d'études immunocytochimiques en double (et même triple) marquage présentés aujourd'hui par Georgio Battaglia et Aldo Rustioni [7] de l'université de Caroline du Nord (USA) remettent en question l'identification de la substance $\mathrm{P}$ comme neurotransmetteur associé aux fibres afférentes nociceptives. Les auteurs ont caractérisé les neurones des ganglions rachidiens, chez le rat et le singe, à l'aide d'anticorps très spécifiques contre la substance $P$ et le glutamate. En réalisant des coupes sériées de seulement $4 \mu \mathrm{m}$ d'épaisseur au travers des ganglions, et en recherchant alternativement la présence de substance $P$ et de glutamate dans les coupes voisines, les auteurs ont démontré que la plupart des neurones qui contiennent de la substance $\mathrm{P}$ - et sont donc des participants présumés des voies de la nociception - contiennent également du glutamate. Après traitement local à la colchicine - qui augmente les concentrations de neurotransmetteur dans le corps cellulaire en interrompant le flux axonal -, près de $90 \%$ des neurones positifs pour la substance $\mathrm{P}$ le sont également pour le glutamate. Or le glutamate est le neurotransmetteur «excitateur» le plus classiquement rencontré dans l'ensemble du système nerveux central !
Le rôle de la substance $\mathrm{P}$ dans la transmission des messages induits par les stimulations nociceptives semble ainsi totalement différent de l'idée que l'on s'en faisait jusqu'alors. Loin d'être le neurotransmetteur spécifique des fibres de la douleur aboutissant dans la moelle épinière, la substance $P$, parce qu'elle est colocalisée avec le glutamate, semblerait bien plus jouer dans les fibres $\mathrm{C}$ un rôle modulateur. Cette activité modulatrice pourrait s'exercer sur l'activité évoquée par le neurotransmetteur classique, comme cela a été démontré dans d'autres systèmes $\left(\mathrm{m} / \mathrm{s} n^{\circ} 1\right.$, vol. 4, p. $57 ; n^{\circ} 4$, vol. 4, p. 255). Quant au VIP présent dans les afférences d'origine viscérale, on pourrait parier - avec beaucoup de chances de gagner - qu'il suffirait de faire la même expérience de double marquage immunocytochimique...

Marc Peschanski

\section{RÉFÉRENCES}

1. Besson JM. La douleur. médecine/sciences 1985 ; 1 : 306-12.

2. Besson JM. Les systèmes de contrôle de la douleur. médecine/sciences $1986 ; 2$ : 493-500. 3. Ruda MA, Bennett GJ, Dubner R. Neurochemistry and neural chemistry in the dorsal horn. In : Emson PC, et al., eds. Peptides and Neurological Diseases. Amsterdam: Elsevier, 1986 : 219-68

4. Henry JL. Effects of substance P on functionally identified units in cat spinal cord. Brain Res 1976 ; 114 : 439-52.

5. Nicoll RA, Schenker C, Leeman SE. Substance $\mathrm{P}$ as a transmitter candidate, Ann Rev Neurosci 1980 ; 3 : 227-68.

6. Basbaum AI, Glaser EJ. Immunoreactive vasoactive intestinal polypeptide is concentrated in the sacral spinal cord: a possible marker for pelvic visceral afferent fibers. Somatosensory Res 1983 ; 1 : 69-82.

7. Battaglia G, Rustioni A. Coexistence of glutamate and substance $P$ in dorsal root ganglion neurons of the rat and monkey. J Comp Neurol 1988 ; 277 : 302-12. 